\title{
Modification of the biopolymer castor oil with free isocyanate groups to be applied as bioadhesive
}

\author{
P. Ferreira ${ }^{a, b, *}$, R. Pereira ${ }^{\text {a }}$, J.F.J. Coelho ${ }^{\text {a }}$, António F.M. Silva ${ }^{\text {a }}$, M.H. Gil ${ }^{a}$ \\ a Departamento de Engenharia Química, Universidade de Coimbra, Polo II, Pinhal de Marrocos, 3030-290 Coimbra, Portugal \\ ${ }^{\mathrm{b}}$ Universidade Católica Portuguesa, Centro Regional das Beiras, Polo de Viseu, Estrada da Circunvalação, 3504-505 Viseu, Portugal
}

Received 24 March 2006; received in revised form 30 June 2006; accepted 30 June 2006

Available online 5 July 2006

\begin{abstract}
Surgical adhesives have been used for several applications, including haemostasis, sealing air leakages and tissue adhesion. The aim of this work was to develop a biodegradable urethane-based bioadhesive containing free isocyanate groups. This material presents the advantage of being biodegradable, biocompatible and having the capacity of reacting with amino groups present in the biological molecules.

A urethane based on castor oil (CO) was synthesized by reaction of the molecule with isophorone diisocyanate (IPD). The characterization of the material was accomplished by different techniques: ATR-FT-IR (attenuated transmittance reflection-Fourier transform infrared), swelling capacity determination, evaluation of the moisture curing kinetics, reaction with aminated substrates and determination of surface energy by contact angle measurement. The study of the urethane thermal properties was performed by DMTA (dynamical mechanical thermal analysis) and TGA (thermogravimetric analysis). The haemocompatibility of the urethane was also evaluated by thrombosis and haemolysis tests.
\end{abstract}

(C) 2006 Elsevier B.V. All rights reserved.

Keywords: Surgical adhesives; Surface energy; Thermal analysis; Haemocompatibility

\section{Introduction}

Primary wound healing of a plan-to-plan oriented scar is usually accomplished by hand sewing or stapling the corresponding layers of each side of the incision [1]. Both these methods have been associated to wound infection. Suture strands can be classified according to their degradation properties as well as to their chemical composition. Natural absorbable suture strands are usually based on collagen and when implanted in living organisms are digested by body enzymes which attack and break down the strand. Synthetic absorbable sutures are based in synthetic polymers. When implanted they are hydrolyzed, resulting in the breakdown of the polymer chain. Hydrolyzation results in a lesser degree of tissue reaction than the enzymatic action suffered by natural absorbable sutures. Suture strands can also be nonabsorbable. These are not digested by body enzymes or hydrolyzed in body tissue and can be made from a variety of nonbiodegradable materials. The response of the organism to its presence involves a process of encapsulation by the body's

\footnotetext{
* Corresponding author. Tel.: +351 239798743; fax: +351 239798703.

E-mail address: paula_calvinho@yahoo.com (P. Ferreira).
}

fibroblasts. Nonabsorbable stands as well as metallic stapes have to be removed when applied in exterior skin closure.

To overcome these limitations surgeons have seeked alternative such as the use of medical tissue adhesives. These adhesives consist of an atractive alternative to suturing or stapling since they exhibit advantageous features, such as haemostasis sealing of air leakages and the elimination of the risk needle-stick injury to the surgeon. The use of an adhesive, being an easier and faster method to establish tissue adhesion, would also reduce the surgical procedure time. However, surgical adhesives must meet some clinical requirements. They must hold the two sides of the tissue together until it has enough mechanical strength to properly support wound healing and then they should be degraded to biocompatible products [2].

Nowadays, the most used surgical adhesives are based on fibrin $[3,4]$ and cyanoacrylates $[5,6]$. The fibrin based adhesives present several problems, e.g. immunogenicity and risk of blood transmitted diseases such as HIV and BSE. On the other hand, cyanoacrylates have been reported to degrade in aqueous media to produce formaldehyde, which causes inflammation and has got carcinogenicity potential. Other options have recently been explored and among the synthetic materials, urethane-based adhesives have been considered to be the most 


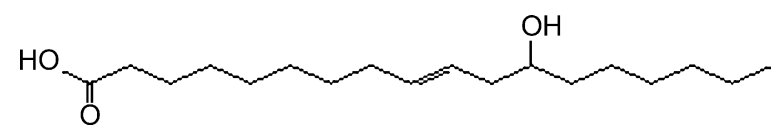

Fig. 1. Representation of the ricinoleic acid structure.

promising [7]. These materials are good candidates due to the possibility of being biodegradable, biocompatible and, if synthesised in the form of prepolymers, have the capacity to react with amino groups present in the biological molecules. This reaction results in the formation of urea linkages and also on the promotion of adhesion since these covalent bonds will hold the tissue together. An adhesive based on polyurethanes was synthesised and studied by Lipatova [8]. These authors showed that their degradation products did not present any toxic effect and that the glue was auto sterile and assured intensive haemostasis. Its potential as surgical adhesive was tested in several applications, such as renal surgery [9], endocrinology [10] and pancreatic occlusion [11]. Another advantage of the urethanes is the possibility of being biodegradable if they are synthesised from e.g. natural molecules, such as castor oil.

Castor oil is clear, almost colourless or pale yellow coloured viscous oil. This compound consists of a triglyceride of fatty acids, where, approximately, $90 \%$ of their content corresponds to the ricinoleic acid (Fig. 1).

This aliphatic carboxylic acid presents an 18-carbon chain, having a double bond between carbon 9 and 10 and a hydroxyl group on the carbon 12. This combination of hydroxyl group and unsaturation occurs exclusively in the castor oil molecule.

Although the plant from which the castor oil is extracted can be found through the world, it is mainly explored in Brazil and India. The composition of this natural oil is, however, extraordinarily well conserved independently of its origin (Fig. 2).

Castor oil is a vegetable oil that has found applications in many chemical industries [12]. When dehydrated, it is converted into a fast drying oil widely applied in paints, varnishes and even adhesives [13]. Its high water resistance makes it ideal for the use in all types of wrappings and packages for the food industry. This oil is also the primary raw material for the production of sebacic acid which is the basic ingredient for nylon production.

In the medical and pharmaceutical field, castor oil is mainly used in hospitals in colon RX patients' preparation since it presents laxative properties. This compound can also be found in several other products such as skin moisturisers (including lotions for solar burnings and dermatitis), cosmetics [14], creams or contraceptive gels, herbal preparations for labour stimulation [15], and systems of drug controlled release [16].

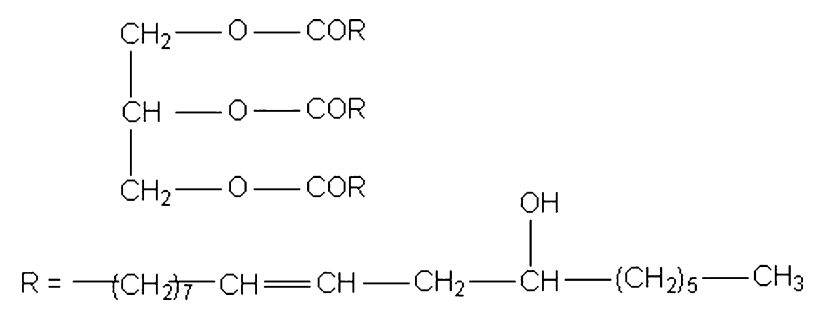

Fig. 2. Fundamental structure of castor oil.
The present paper describes the synthesis of a urethane based on castor oil in order to obtain a prepolymer with terminal isocyanate groups. The characterization of the urethane was accomplished by different techniques: ATR-FT-IR (attenuated total reflectance-Fourier transform infrared), swelling capacity determination, study of the stability of NCO groups under humidity conditions, reaction with aminated substrates and determination of surface energy by contact angle measurement. Thermal properties were also evaluated by DMTA and TGA. The haemocompatibility of the urethane was also evaluated by thrombosis and haemolysis tests.

\section{Experimental procedure}

\subsection{Materials}

All the reagents were purchased from Sigma/Aldrich Chemical Company (Spain) and used with no further treatment. Rabbit venous blood used in haemocompatibility studies was colleted in polypropylene tubes with a 9:1 blood ACD (acid citrate dextrose) solution [17] ratio and was used immediately after collection.

\subsection{Synthesis}

Urethanes based on castor oil were synthesized by modification of their hydroxyl groups with isophorone diisocyanate (IPD, Fig. 3). The ratio of NCO:OH groups used was 2:1.

The reactions were performed by stirring the two components at $60^{\circ} \mathrm{C}$ under a nitrogen atmosphere. By ATR-FT-IR technique it was possible to observe that after $24 \mathrm{~h}$ of reaction, all the CO hydroxyl groups had reacted with the NCO groups of the isocyanate resulting in the formation of urethane groups. This technique was also employed to confirm the presence of free isocyanate groups in the prepolymer. All the ATR-FT-IR analysis were performed on a Magma-IR ${ }^{\mathrm{TM}}$ Spectrometer 750 from Nicolet Instrument Corp., equipped with a Golden Gate Single Reflection Diamond ATR. Spectra were recorded on an average of 128 scans at a resolution of $4 \mathrm{~cm}^{-1}$.

\subsection{Swelling, moisture curing kinetics and reaction with aminated substrates}

The moisture curing kinetics was determined by evaluating the stability of the NCO groups present in the prepolymers when

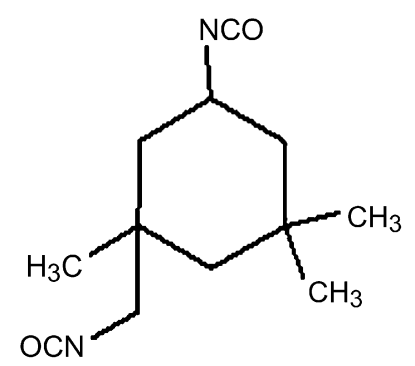

Fig. 3. Structure of isophorone diisocyanate (IPD) used to synthesize the urethanes. 


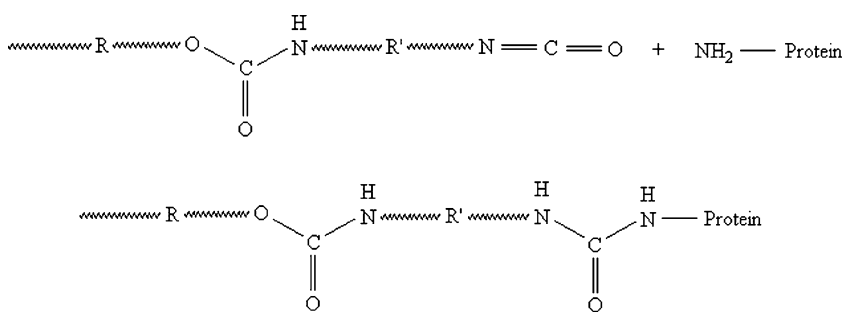

Fig. 4. Reaction between a prepolymer and the amino groups of a protein resulting in a urea linkage.

the samples were kept in a sealed container and submitted to a water saturated atmosphere. This parameter was evaluated by measuring the length of the band at $2250 \mathrm{~cm}^{-1}$, which corresponds to the NCO free groups, by the ATR-FT-IR technique until the band was no longer detectable.

The water sorption capacity of the samples was also evaluated. The weight of the dried samples was obtained $\left(W_{\mathrm{d}}\right)$. These samples were then placed in a container with a saturated solution of pentahydrated copper sulphate and were weighted at different times until a maximum weight was achieved $\left(W_{\mathrm{s}}\right)$. The swelling ratio was evaluated by using Eq. (1):

swelling ratio $(\%)=\left(\frac{W_{\mathrm{s}}-W_{\mathrm{d}}}{W_{\mathrm{d}}}\right) \times 100$

One of the advantages of urethane prepolymers as bioadhesives is their ability to react with the amino groups of the proteins existent in the living tissues establishing bonds between them in the form of urea linkages. These reactions, combined with moisture curing of the adhesive (Section 3.2) result in tissue adhesion (Fig. 4).

In order to evaluate the adhesive capacity of the prepolymers, they were placed between gelatine sheets that present a great amount of amino groups and therefore simulate the living tissues. The tests were performed with gelatine sheets which were either dry, moisturised with water or moisturised with plasma. The sheets were then subjected to a binding strength test, using a Chatillon TCD 1000 . The pulling velocity was $20 \mathrm{~mm} / \mathrm{min}$, and the assays were carried out at room temperature. The software program coupled to the apparatus registered the applied force and length variation. The tests terminated with the fracture of the gelatine sheets or their separation in case adhesion failed to occur.

\subsection{Determination of surface energy by contact angle measurement}

It is widely recognised that surface energy is an important parameter affecting polymers adhesion, material wettability and even biocompatibility [18].

The measurement of contact angles is considered the most convenient method for determining the surface free energy of solid samples. This technique relies on the determination of the interactions between the solid sample of interest and liquids with well determined surface tensions (Fig. 5).

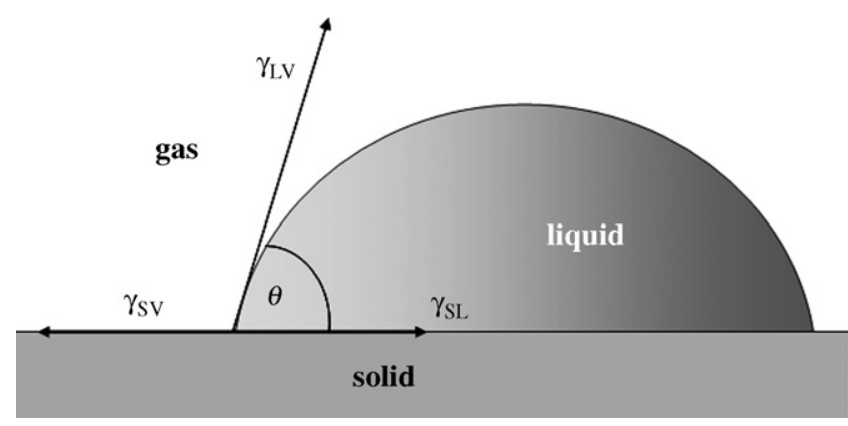

Fig. 5. Scheme of a drop on the surface of a solid.

The equilibrium of forces at the edge of a resting drop can be described by Young's equation $[19,20]$ (Eq. (2)):

$\gamma_{\mathrm{S}}=\gamma_{\mathrm{SL}}+\gamma_{\mathrm{L}} \cos \theta$

In Eq. (2), $\gamma_{S}$ and $\gamma_{\mathrm{L}}$ represent respectively the surface free energy of the solid and the surface tension of probe liquid. $\gamma_{\mathrm{SL}}$ corresponds to their common interfacial free energy and $\theta$ the equilibrium contact angle [19,20].

According to Owens, Wendt, Rabel and Kaelble the interfacial tension can be divided in two components: dispersive interactions and polar interactions [21]. Polar interactions contain Coulomb interactions between permanent dipoles and the ones between permanent and induced dipoles. The interactions caused by time fluctuations of the charge distribution within the molecules are called dispersive interactions:

$\gamma_{\mathrm{S}}=\gamma_{\mathrm{S}}^{\mathrm{D}}+\gamma_{\mathrm{S}}^{\mathrm{P}}, \quad \gamma_{\mathrm{L}}=\gamma_{\mathrm{L}}^{\mathrm{D}}+\gamma_{\mathrm{L}}^{\mathrm{P}}$

$\gamma_{\mathrm{SL}}=\gamma_{\mathrm{S}}+\gamma_{\mathrm{L}}-2\left(\sqrt{\gamma_{\mathrm{S}}^{\mathrm{D}} \gamma_{\mathrm{L}}^{\mathrm{D}}}+\sqrt{\gamma_{\mathrm{S}}^{\mathrm{P}} \gamma_{\mathrm{L}}^{\mathrm{P}}}\right)$

Substituting this term for $\gamma_{\mathrm{SL}}$ in Eq. (2) results:

$\gamma_{\mathrm{S}}=\gamma_{\mathrm{S}}+\gamma_{\mathrm{L}}-2\left(\sqrt{\gamma_{\mathrm{S}}^{\mathrm{D}} \gamma_{\mathrm{L}}^{\mathrm{D}}}+\sqrt{\gamma_{\mathrm{S}}^{\mathrm{P}} \gamma_{\mathrm{L}}^{\mathrm{P}}}\right)+\gamma_{\mathrm{L}} \cos \theta$

Finally it is possible to obtain an equation of a straight line of the form

$y=a x+b$

where

$y=\frac{1+\cos \theta}{2} \frac{\gamma_{\mathrm{L}}}{\sqrt{\gamma_{\mathrm{L}}^{\mathrm{D}}}}, \quad a=\sqrt{\gamma_{\mathrm{S}}^{\mathrm{P}}}, \quad x=\sqrt{\frac{\gamma_{\mathrm{L}}^{\mathrm{P}}}{\gamma_{\mathrm{L}}^{\mathrm{D}}}}, \quad b=\sqrt{\gamma_{\mathrm{S}}^{\mathrm{D}}}$

Plotting $y$ versus $x$ it becomes possible to calculate $\gamma_{\mathrm{S}}^{\mathrm{P}}$ and $\gamma_{\mathrm{S}}^{\mathrm{D}}$, and the sum of both contributions establishes the value of surface free energy.

During this work, surface energies of the urethane and gelatine sheets were evaluated, by static contact angle $(\theta)$ measurements in an OCA 20 from Dataphysics in order to compare them with the ones obtained from literature for skin and blood tissues. All the tests were performed on the air-facing surfaces of the samples with four liquids: water, formamide, ethylene glycol and propylene glycol using the sessile drop method. Nine 
measurements on different points were performed to calculate the mean static contact angle $\theta$ and its standard deviation.

The dispersive $\gamma_{\mathrm{S}}^{\mathrm{D}}$ and polar $\gamma_{\mathrm{S}}^{\mathrm{P}}$ components of the urethane as well as the one of the gelatine sheets were determined according to the Owens-Wendt-Rabel and Kaelble relationship.

\subsection{Thermal properties}

Thermal properties were evaluated both to the urethane and unmodified CO. Thick specimens $(15.20 \mathrm{~mm} \times 7.45 \mathrm{~mm} \times$ $1.10 \mathrm{~mm}$ ) were analysed by dynamical mechanical thermal analysis (DMTA). A Triton Tritec 2000 analyser was used in the constrain layer damping mode, with a standard heating rate of $2{ }^{\circ} \mathrm{Cmin}^{-1}$, in muiltifrequencies mode $(1,3$, and $10 \mathrm{~Hz})$ and a displacement of $0.05 \mathrm{~mm}$. The glass transition temperature $\left(T_{\mathrm{g}}\right)$ was determined as the peak in $\tan \delta\left(\tan \delta=E^{\prime \prime} / E^{\prime}\right)$ where $E^{\prime \prime}$ and $E^{\prime}$ are the loss and storage modulus, respectively, derived from DMTA. The TGA (thermogravimetric analysis) curves were also obtained at a heating rate of $2^{\circ} \mathrm{C} \mathrm{min}^{-1}$ in a SDT Q600 from thermal analysis.

\subsection{Haemocompatibility}

The haemocompatibility was evaluated in vitro according to the International Standard Organization (ISO) 10993-4 [22], and the following categories of blood interactions were studied: thrombogenicity and haemolysis.

\subsubsection{Thrombogenicity}

The gravimetric method of Imai and Nose [23] was followed to evaluate thrombus formation on three samples of polymeric surfaces. For that purpose, anticoagulated rabbit blood (ACD blood) was prepared by adding $1 \mathrm{~mL}$ of ACD solution to $9 \mathrm{~mL}$ of fresh rabbit blood. Before performing the tests, the urethanes were kept in contact with PBS (phosphate buffered saline $\mathrm{pH}$ 7.4) at a constant temperature of $37^{\circ} \mathrm{C}$. After $24 \mathrm{~h}$ of incubation, the PBS was removed and the ACD blood was added to the polymers and also to an empty Petri dish (positive control). Blood clotting tests were initiated by adding $0.02 \mathrm{~mL}$ of a $0,10 \mathrm{M}$ calcium chloride solution and were stopped by the addition of $5 \mathrm{~mL}$ of water after $45 \mathrm{~min}$. The resultant clots were fixed with $5 \mathrm{~mL}$ of a formaldehyde solution $36 \%$ and were then dried with tissue paper and finally weighted.

\subsubsection{Haemolysis}

The haemolysis tests were performed as described in American Society for Testing and Materials (ASTM) F 756-00 standard [24].

Three samples of each urethane $\left(21 \mathrm{~cm}^{2}\right)$ were placed in polypropylene test tubes and $7 \mathrm{~mL}$ of PBS were added. After $72 \mathrm{~h}$ of incubation at $37^{\circ} \mathrm{C}$, the PBS was removed and $1 \mathrm{~mL}$ of diluted ACD rabbit venous blood $(9.02 \mathrm{mg} / \mathrm{mL})$ was added to each sample. ACD blood was also added both to the PBS extraction solution and to three samples of the urethanes with no previous treatment with PBS and they were all maintained at $37^{\circ} \mathrm{C}$ for $3 \mathrm{~h}$. Positive and negative controls were prepared by adding the same amount of ACD blood to $7 \mathrm{~mL}$ of water and PBS, respectively. Each tube was gently inverted twice each 30 min to maintain contact of the blood with the material. After incubation, each fluid was transferred to a suitable tube and centrifuged at $2000 \mathrm{rpm}$ for $15 \mathrm{~min}$. The haemoglobin released by haemolysis was measured by the optical densities (OD) of the supernatants at $540 \mathrm{~nm}$ using a spectrophotometer UV-vis (Jasco V-550). The percentage of haemolysis was calculated as described in the following equation:

$$
\text { Haemolysis }(\%)=100\left(\frac{\mathrm{OD}_{\text {test }}-\mathrm{OD}_{\text {negative control }}}{\mathrm{OD}_{\text {positive control }}-\mathrm{OD}_{\text {negative control }}}\right)
$$

\section{Results and discussion}

\subsection{Synthesis}

A urethane prepolymer was synthesized by reacting $\mathrm{CO}$ with isophorone diisocyanate (IPD). The urethane was prepared in a $2 / 1$ ratio of $\mathrm{NCO} / \mathrm{OH}$ groups to ensure that some terminal isocyanate groups remained free (Fig. 6).

The reactions were confirmed by ATR-FT-IR by detection of the urethane band at $1515 \mathrm{~cm}^{-1}$. It was also determined that all the CO hydroxyl groups had reacted with the NCO groups of the isocyanate since the broad band that corresponded to the $\mathrm{OH}$ groups $\left(3401 \mathrm{~cm}^{-1}\right)$ was no longer detected in the urethanes spectra.

The presence of the NCO free groups was also shown by the same technique, since it was possible to detect a very intense band at $2250 \mathrm{~cm}^{-1}$, which corresponds to the existence of these groups. The obtained ATR-FT-IR spectra for CO and for the urethane CO-IPD are presented in Fig. 7.

\subsection{Swelling, moisture curing kinetics and reaction with aminated substrates}

Wounds such as surgical ones which result in a limited loss of tissue usually heal rapidly on the surface. This scarring process proceeds as the opposing edges of the wound come in close proximity so the cellular repair mechanism can take place. If a polymer swells two much when in contact with living tissues, this may mean that it will damage the surrounding tissues due to an increase in its volume. This phenomenon can lead to a deficient healing process and possible secondary effects such as infections and scaring. However, the castor oil molecule presents a very hydrophobic nature and, for that reason, there was no variation in urethanes weight and volume trough time, which means that the swelling was $0 \%$ and that these damaging effects will not occur with the application of this material.

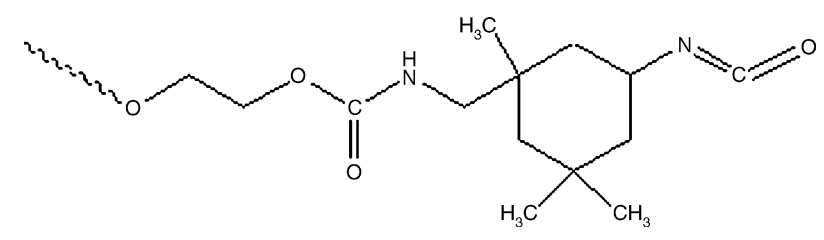

Fig. 6. Urethane with terminal NCO groups. 


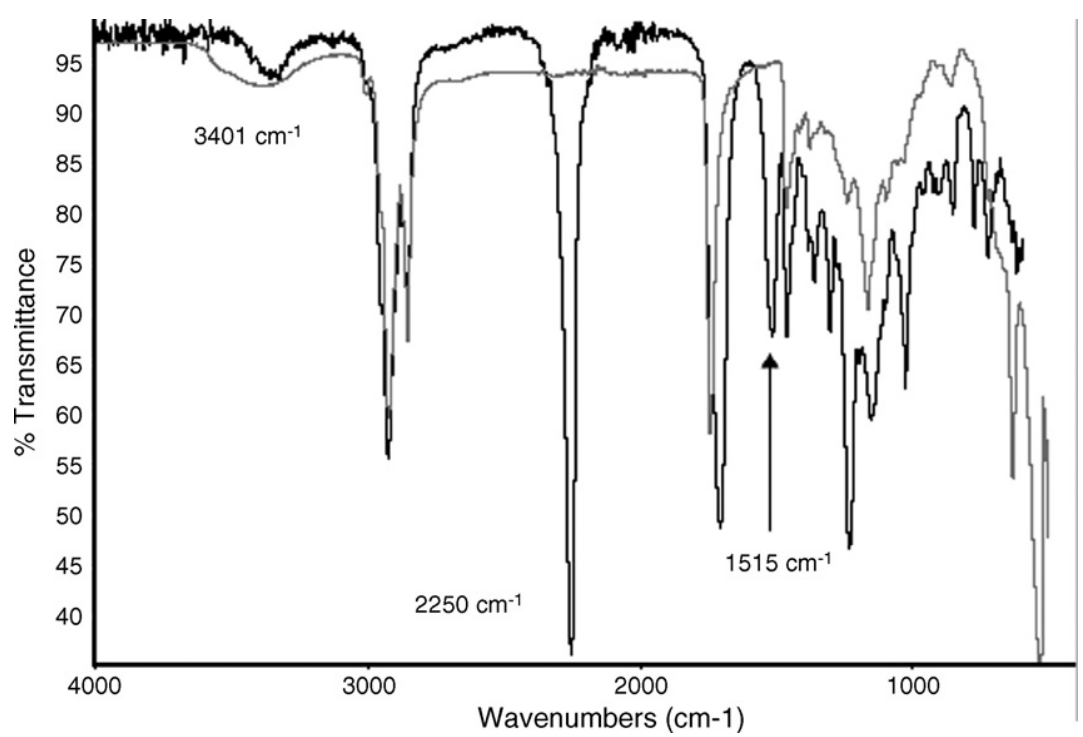

Fig. 7. ATR-FT-IR spectrum obtained for the $\mathrm{CO}$ and CO-IPD urethane. The gray line represents the spectrum of $\mathrm{CO}$ and the black line represents the spectrum of CO-IPD.

As stated before, these prepolymers present the ability to favour the adhesion of the living tissues due to the reaction with the amino groups of their proteins. Therefore, the importance of analysing the stability of NCO free groups becomes evident, even in extreme conditions.

The isocyanate end groups present in this type of polymers are also able to react with air moisture resulting in the formation of carbon dioxide and an aminic ending polymer [25].

However, further reactions may occur, resulting, in a first step, in the formation of urea groups. Finally, the isocyanate groups may react with these urea units and, consequently, the adhesive which was firstly linear becomes crosslinked (Fig. 8) [25].

Although this moisture curing of the adhesive will necessarily occur in the living tissues, it is also important to avoid this occurrence while the adhesive is still not being used, during manipulation or even in storage.

The stability of the NCO groups, measured by the air moisture curing kinetics, was evaluated by placing the samples in a

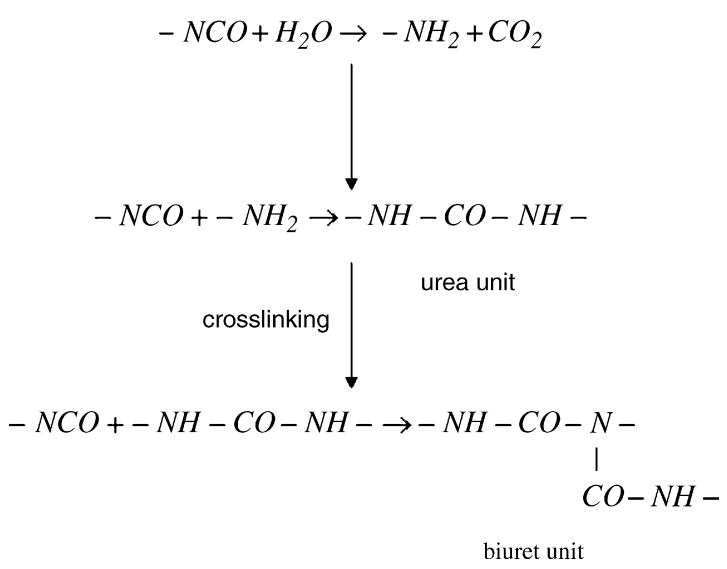

Fig. 8. Reaction between a isocyanate groups and water resulting in the formation of aminic groups and finally in the crosslinking of the adhesive. water saturated atmosphere and following the evolution of the $2250 \mathrm{~cm}^{-1}$ band length by the ATR-FT-IR technique, which corresponds to the NCO free groups. The results obtained are presented in Fig. 9.

When analysing the chart, we can verify that after one day of exposure no statistical differences were observed in the amounts of NCO free groups still present in the samples. This can be explained by the hydrophobicity of the $\mathrm{CO}$ molecule. On the second day, $10 \%$ of the NCO groups were already hydrolysed and on the fifth day that percentage raised to $61 \%$. The total hydrolysis of the isocyanate groups occurred when 7 days were completed. Considering however that the tests were performed in a water saturated atmosphere, the NCO groups seem to be stable enough to be kept in storage conditions as long as humidity is avoided until its application.

When considering the application conditions, it is important to keep in mind that water will be present in the living tissues. It is known, however, that the isocyanate reactivity with water and primary hydroxyl group is comparable, but much lower than with amines [26] (Table 1).

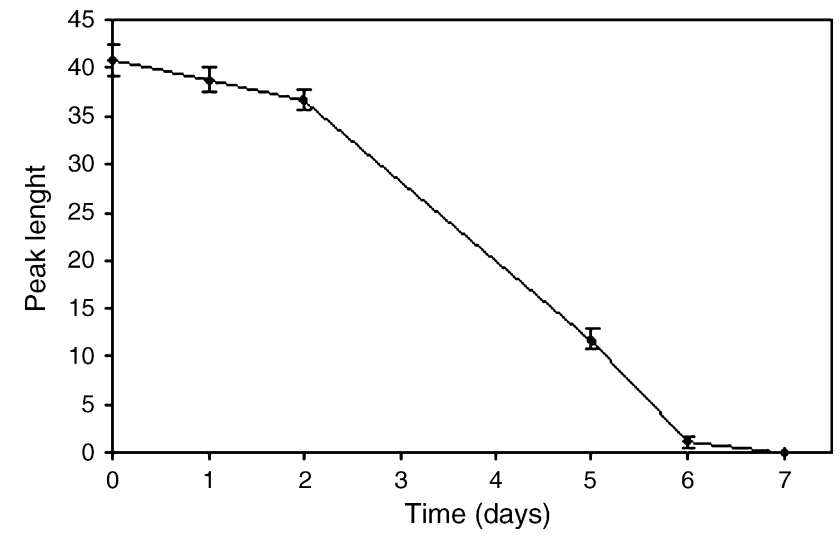

Fig. 9. Evolution of the peak length corresponding to the NCO free groups. 
Table 1

Relative reactivity of isocyanates with hydrogenated compounds

\begin{tabular}{lll}
\hline Hydrogenated compound & General structure & $\begin{array}{l}\text { Relative reaction rate (at } \\
25^{\circ} \mathrm{C} \text { and without catalyst) }\end{array}$ \\
\hline Primary aliphatic amine & $\mathrm{R}^{-N_{2}}$ & 100,000 \\
Secondary aliphatic amine & $\mathrm{RR}^{\prime} \mathrm{NH}$ & $20,000-50,000$ \\
Primary hydroxyl & $\mathrm{RCH}_{2}-\mathrm{OH}$ & 100 \\
Water & $\mathrm{HOH}$ & 100 \\
Secondary hydroxyl & $\mathrm{RR}^{\prime} \mathrm{CH}-\mathrm{OH}$ & 30 \\
Tertiary hydroxyl & $\mathrm{RR}^{\prime} \mathrm{R}^{\prime \prime} \mathrm{C}-\mathrm{OH}$ & 0.5 \\
Amide & $\mathrm{RCO}^{-\mathrm{NH}_{2}}$ & 0.1 \\
\hline
\end{tabular}

Considering this, it is expected that the reaction between $\mathrm{NCO}$ and amino groups in the living tissues occurs much faster than the one between NCO and water although it will be unavoidable that some does happen. In fact, the presence of humidity will be an important parameter in adhesion efficacy considering that curing of this type of prepolymers occurs in the presence of water. This statement was also confirmed by the results obtained during reaction with aminated substrates.

The binding capacity of the adhesive was determined by placing them between dry, moisturised with water and moisturised with plasma gelatine sheets. These were then subjected to the binding strength test which terminated either with the fracture of the gelatine sheets or the separation of them if adhesion failed to occur. The values of maximum force $(\mathrm{N})$ were registered and are presented in Fig. 10.

When analysing the chart represented in Fig. 10 one can observe that the urethane was unable of gluing the gelatine sheets in the dry state. In fact, urethanes prepolymers are adhesives which are cured by moisture either from air or from substrate, and the mechanism consists in a condensation polymerization [27]. This is why, when moisture was present, the values of force were much higher and in this case the gelatine sheets were efficiently glued and were only separated at breaking point. This higher efficiency of the adhesive in the presence of an aqueous medium is explained by the reactions presented in Fig. 8. Throughout these reactions, the adhesive which was linear becomes crosslinked, or in other words, curing of the adhesive occurs. For this reason we can conclude that the presence of moisture in the living tissues will in fact be an important factor in the efficacy of the adhesive once applied.

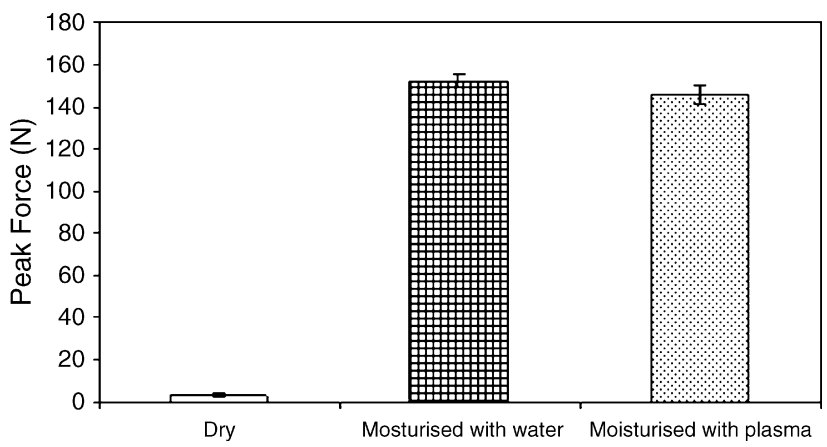

Fig. 10. Values of peak force for each polyurethane at dry and moisturised conditions.

\subsection{Determination of surface energy by contact angle measurement}

For any adhesive to adhere to a substrate, one fundamental thermodynamic requirement has to be satisfied first: the measured surface energy of the adhesive must be equal to or less than that of the adherent. Unless this condition is satisfied, a material cannot adhere to the substrate [28].

The main purpose of this measurement was to evaluate how well the adhesive would spread when placed over the aminated substrate, over skin and over a bleeding surface. For skin, the critical surface energy varies between 38 and $56 \mathrm{mN} / \mathrm{m}$ depending on the temperature and relative humidity of the skin [28]. The value for surface tension of blood is $47.5 \mathrm{mN} / \mathrm{m}$ and was assessed by Agathopoulos and Nikolopoulos [29] by the sessile drop method at a temperature of $37^{\circ} \mathrm{C}$.

In order to evaluate this parameter, surface tensions of the urethane and gelatine sheets were determined, by static contact angle $(\theta)$ measurements with four liquids: water, formamide, ethylene glycol and propylene glycol. According to the OwensWendt-Rabel and Kaelble relationship, the dispersive $\gamma_{\mathrm{S}}^{\mathrm{D}}$ and polar $\gamma_{\mathrm{S}}^{\mathrm{P}}$ components of the urethane as well as the ones of the gelatine sheets were determined. The obtained results as well as the ones for skin and blood are presented in Table 2.

The results showed that the surface energy of gelatine, skin and blood are much higher than the ones of the urethane.

This means that the forces between the molecules that make up the urethane are weak and consequently adhesion between them and any of these surfaces is therefore likely to happen. The adhesive forces between any of these surfaces and the urethane will overcome the cohesive forces of this prepolymer and intermolecular proximity between the adhesive and the surface will therefore occur.

\subsection{Thermal properties}

Dynamic mechanical thermal analysis is an extremely versatile and sensitive method which enables the complete scan of relaxation mechanisms in viscoelastic materials. This equipment has been intensely used and recognized as a powerful tool to identify the materials thermal transitions due to its extremely high sensitiveness. Its special sensibility to determine frequency dependent transitions such as the glass transition temperature that represents the motions of long chain segments is of outstanding importance. The basic function of a DMTA is to obtain information about the mechanical and thermal properties of the materials by applying a sinusoidal load to a specimen and mea-

Table 2

Surface energy values and correspondent dispersive and polar components

\begin{tabular}{llcc}
\hline Substrate & \multicolumn{3}{l}{ Surface energies and surface tensions $(\mathrm{mN} / \mathrm{m})$} \\
\cline { 2 - 4 } & $\sigma_{\mathrm{S}}$ & $\sigma_{\mathrm{S}}^{\mathrm{D}}$ & $\sigma_{\mathrm{S}}^{\mathrm{P}}$ \\
\hline Gelatine & 44.24 & 5.00 & 39.24 \\
Skin [28] & $38-56$ & - & - \\
Blood [29] & 47.5 & 11.2 & 36.3 \\
PU CO & 14.87 & 9.35 & 5.52
\end{tabular}




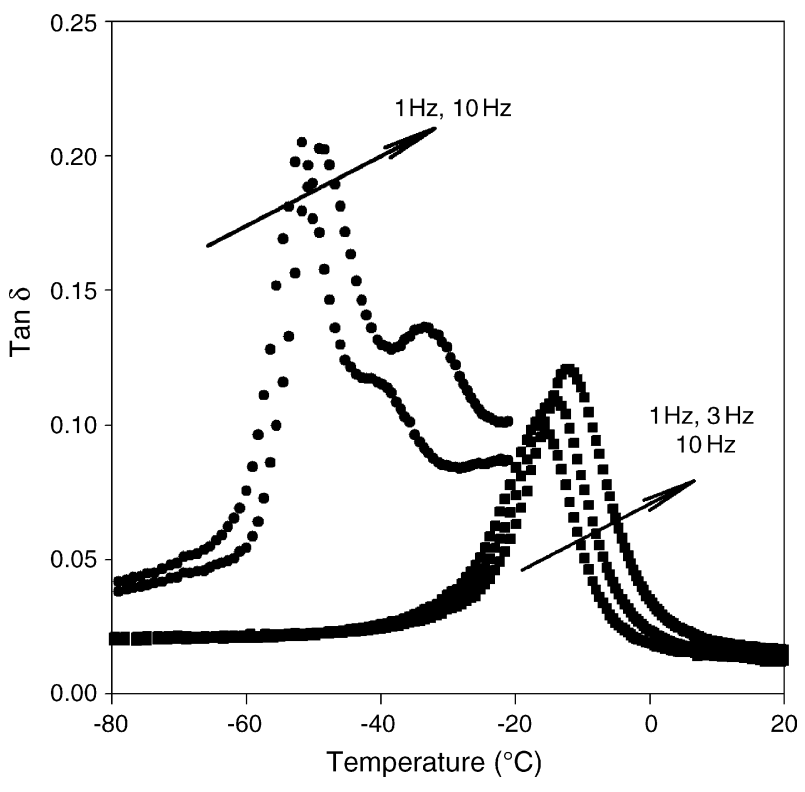

Fig. 11. Multifrequency $\tan \delta$ traces obtained for:

suring the resultant deformation, while the sample is subjected to a controlled temperature programme. The load depends on the material and sample geometry, but normally is set at very low values which make it possible to detect small changes in the mechanical and thermal behaviour of the material. In the scope of this work, the DMTA data were used to identify the thermal transitions of CO due to the modification with IPD.

The DMTA was carried out in the Constrain Layer Damping Mode, following a methodology previously used by the same authors for different materials with high degree of success [30-33]. Fig. 11 shows the DMTA traces in multifrequency mode of the raw material used, in this case the $\mathrm{CO}$, and the product obtained by reaction with IPD.

The dynamic mechanical $\tan \delta$ peak is expected to shift to higher temperatures with increasing frequency for any thermally activated relaxation process as shown in Fig. 11.

Among other advantages, the utilization of multifrequency analysis in DMTA runs allows the user to distinguish between the frequency dependent process such as the molecular relaxations ( $\alpha$ and $\beta$ transitions) and those that are not frequency dependent such as melting, curing, crystallization and thermal degradation.

The apparent activation energy $(\Delta H)$ for $\alpha$-relaxation can be determined form the Arrhenius rate relationship:

$\ln f=-\left(\frac{\Delta H}{R T}\right)$

where $f$ is the frequency of analysis, $R$ the universal gas constant, $\Delta H$ the activation energy and $T$ is the temperature in Kelvin.

The $\tan \delta$ of the $\mathrm{CO}$ shows a wide broad peak with two relaxation transitions that are dependent on the frequency. This result is a clear indication of the heterogeneity of this compound, which was previously discussed in the introduction. On the contrary, the $\tan \delta$ of the reaction product between the CO and IPD shows a single narrow smooth peak, suggesting the success of the reaction and the absence of any microphase separation. The $T_{\mathrm{g}}$ value

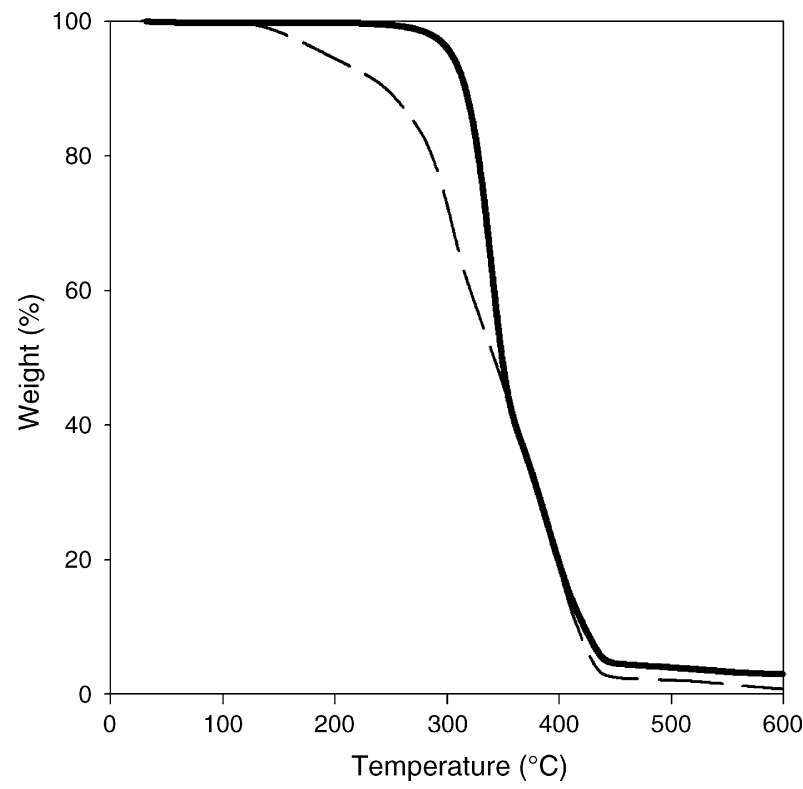

Fig. 12. Thermogravimetric traces obtained for CO (filled line) and CO-IPD (dotted line) at a heating rate of $2{ }^{\circ} \mathrm{C} \mathrm{min}^{-1}$.

obtained is around $-16.9^{\circ} \mathrm{C}$ which corresponds to a flexible material at room temperature.

Furthermore, it is possible to observe a complete movement of the $\tan \delta$ peak of the $\mathrm{CO}$ towards high temperatures. This observation allows to state that the entire $\mathrm{CO}$ reacted with IPD suggesting a nearly $100 \%$ reaction yield. The apparent energy for the $\alpha$-relaxation of the new material is $111 \mathrm{~kJ} / \mathrm{mol}$. This value is quite similar to that obtained $(108 \mathrm{~kJ} / \mathrm{mol})$ in the same laboratory for a material prepared with poly(ethylene glycol) (PEG) modified with IPD [34], and to be used in the same final application. In spite of this similarity, which indicates a close energy involved in the $T_{\mathrm{g}}$ transition for both materials, it should be stressed that the material proposed in this publication is more advantageous due to the higher biodegradability and biocompatibility resulting from the natural source of the CO. In addition, the DMTA trace show a decrease of the $\tan \delta$ values after the reaction, which indicates an increase of material elastic response compared to CO alone.

The thermogravimetric traces presented in Fig. 12, suggest that the onset of mass loss is around 298 and $139^{\circ} \mathrm{C}$ for the $\mathrm{CO}$ and CO-IPD, respectively.

The former value is relatively close to the boiling point of $\mathrm{CO}\left(313^{\circ} \mathrm{C}\right.$, according to the supplier). Concerning the new material proposed in this work, the decomposition temperature determined shows that no problems should be expected when applied at physiologic temperature $\left(\approx 37^{\circ} \mathrm{C}\right)$.

\subsection{Haemocompatibility}

\subsubsection{Thrombogenicity}

The capacity of thrombus formation of three samples of urethanes was evaluated by a gravimetric method. The weights of the blood clots formed after $45 \mathrm{~min}$ are presented in Fig. 13.

It was observed that clot formation was higher for the urethane than for the control. Considering that glass, which con- 


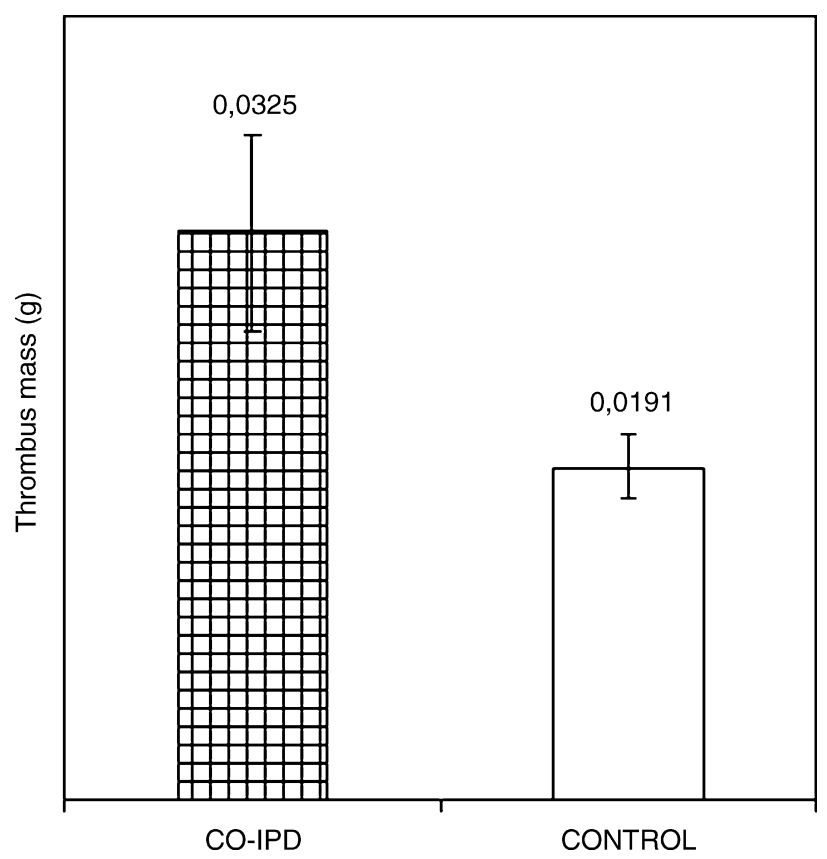

Fig. 13. Weight of thrombus (g) formed after $45 \mathrm{~min}$ of contact with blood.

stituted the positive control is a highly thrombogenic material, it can be concluded that the urethane was thrombogenic. This characteristic is directly related to the low surface energy presented by the polymer. Surface energy can be understood as a measure of hydrophobicity/hydrophilicity. High surface energy means that the surface is hydrophilic, and low surface energy characterizes the surfaces as hydrophobic. At low surface energy materials, proteins adsorb strongly and irreversibly and at high surface energy materials, proteins adsorb weakly and reversibly [35]. Since the protein adhesion constitutes the first step to initiate the coagulation cascade which ends in thrombus formation, it was predictable that the urethanes would present a high thrombogenic character. For the majority of the biomaterials, especially the ones designed to act as implants this would be an undesirable feature. However, considering that the adhesive would be applied in diffuse surfaces with capillary bleeding, its haemostatic character can be of a great importance. Although it will probably not be able to stop active arterial bleeding we can suggest that this material would potently act as a haemostatic agent, amplifying the in vivo thrombogenic potential, improving coagulation, and therefore help the cicatrisation process of the wound.

\subsubsection{Haemolysis}

During haemolysis assay, three samples of test and control materials were placed in contact with ACD rabbit blood under identical conditions and the increase in released haemoglobin was measured.

Values of haemolysis were obtained for samples contacting directly with blood, samples which were kept with PBS pH 7.4 solution for $24 \mathrm{~h}$ at $37^{\circ} \mathrm{C}$ and also for the PBS solution in contact with them (Fig. 14).

According to ASTM F 756-00 [24] materials can be classified as follows (Table 3).

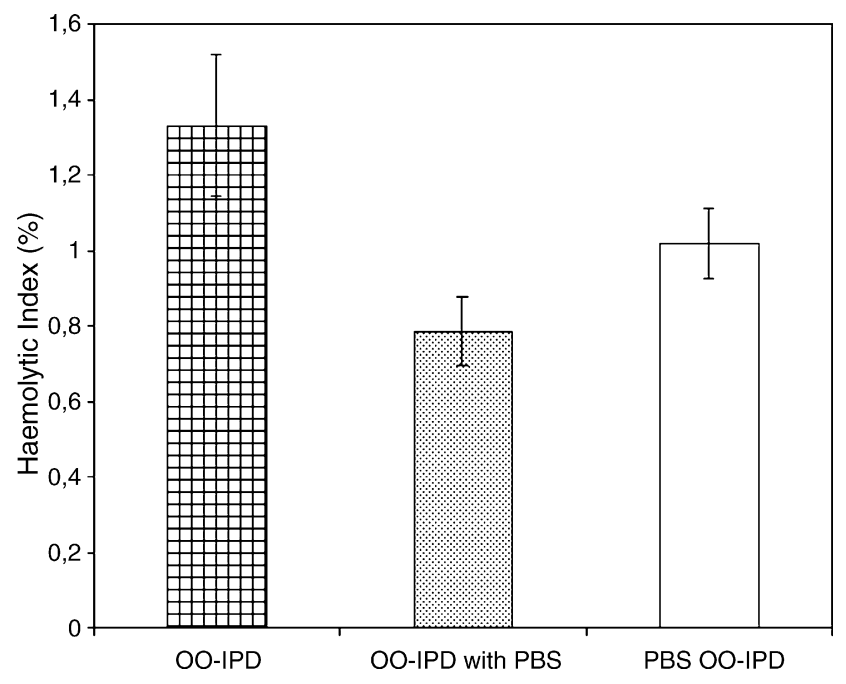

Fig. 14. Values of haemolysis of the urethanes not subjected to extraction, the urethanes incubated in PBS and of the PBS extraction solution.

Table 3

Classification of haemolytic grade of samples according to their haemolysis (\%)

\begin{tabular}{ll}
\hline Haemolysis (\%) above negative control & Haemolytic grade \\
\hline $0-2$ & Non-haemolytic \\
$2-5$ & Slightly haemolytic \\
$>5$ & Haemolytic \\
\hline
\end{tabular}

The synthesised urethanes can be classified as nonhaemolytic, since its haemolytic index is lower than $2 \%$ even when we consider the possible error deviation. As expected in this case, nor the PBS extraction solution or the samples which were kept with PBS pH 7.4 solution for $24 \mathrm{~h}$ at $37^{\circ} \mathrm{C}$ presented haemolytic character.

Haemolysis is regarded as an especially significant screening test, since it provides quantification of small levels of plasma haemoglobin which may not be measurable under in vivo conditions. As reported on literature [22], it is not possible to define a universal level of acceptable or unacceptable amounts of haemolysis. Although by definition a blood-compatible material should be non-haemolytic, the truth is that in practice several medical devices cause haemolysis. This means that when such haemolytic effect takes place, it is important to make sure that clinical benefits overcome the risks and that the values of haemolysis are within acceptable limits. In this case such concern does not exist.

\section{Conclusions}

The present paper describes the synthesis of urethanes based on castor oil in order to obtain a prepolymer with terminal isocyanate groups. In order to obtain these prepolymers castor oil hydroxyl groups were modified with isophorone diisocyanate. The polymers were prepared in a 2:1 ratio of $\mathrm{NCO}: \mathrm{OH}$ in order to end up with terminal isocyanate groups. The occurrence of the synthesis reaction was confirmed by ATR-FT-IR. The urethanes exhibited high hydrophobicity, so the effect of damaging the surrounding tissues due to the increase in its volume will not 
be considerable. Also the NCO groups were found to be stable enough to be kept under storage conditions as long as humidity is avoided. Even in water saturated conditions it took 7 days for the hydrolysis of all the NCO groups occurred.

The thermal analysis of the material allowed us to confirm the reaction between $\mathrm{CO}$ and IPD and also that no traces of unreacted $\mathrm{CO}$ was detected in the urethane analysis. It is also possible to say that the urethane is thermally stable at physiological temperature since its degradation started only at $139^{\circ} \mathrm{C}$.

The surface energy of the urethane was proved to be low, which was consistent with the high thrombosis values it induced. Considering that the application of the synthesised material is focused on bleeding conditions, its haemostatic potential will be an advantage. When haemolysis tests were performed, it was concluded that the urethanes presented a non-haemolytic character, even when no extraction with PBS was performed.

\section{Acknowledgement}

The authors would like to thank Fundação para a Ciência e Tecnologia for the financial support to P. Ferreira (SFRH/BD/ 1052/2000).

\section{References}

[1] N. Sheikh, A.A. Katbab, H. Mirzadeh, Int. J. Adhesion Adhesives 20 (2000) 299.

[2] L. Montanaro, C.R. Arciola, E. Cenni, G. Ciapetti, F. Savioli, F. Filippini, L.A. Barsanti, Biomaterials 22 (2001) 59.

[3] C.J. Dunn, K.L. Goa, Drugs 58 (1999) 863

[4] F.H. Silver, M. Wang, G.D. Pins, Biomaterials 16 (1995) 891.

[5] M.E. King, A.Y. Kinney, Nurse Pract. 24 (1999), 66, 69-70, 73-74.

[6] A.B. Leahey, J.D. Gottsch, W.J. Stark, Ophthalmology 100 (1993) 173.

[7] B. Masa, P. Cefelin, T.E. Lipatova, L.A. Bakalo, G.G. Lugovskaya, J. Polym. Sci. Polym. Symp. 66 (1979) 259

[8] T.E. Lipatova, Adv. Polym. Sci. 79 (1986) 65

[9] A.T. Nosov, A.A. Poliakov, Klin Khir 12 (1980) 16.

[10] I.V. Komissarenko, I.M. Kebuladze, A.G. Lysenko, T.V. Shumova, Klin Khir 12 (1985) 19.
[11] V.S. Zemskov, V.I. Biletskii, S.N. Panchenko, V.S. Shchitov, V.N. Blagodarov, Klin Khir 11 (1986) 3.

[12] D.S. Ogunniyi, Bioresource Technology 97 (2006) 1086.

[13] K.P. Somani, S.S. Kansara, N.K. Patel, A.K. Rakshit, Int. J. Adhesion Adhesives 23 (2003) 269.

[14] S. Yabea, T. Sato, J. Solid State Chem. 171 (2003) 7.

[15] B.L. McFarlin, M.H. Gibson, J. O’Rear, P. Harman, J. Nurse-Midwifery 44 (1999) 3.

[16] S. Eiamtrakarn, Y. Itoh, J. Kishimoto, Y. Yoshikawa, N. Shibata, M. Murakami, K. Takada, Biomaterials 23 (2002) 145.

[17] Anticoagulant Citrate Dextrose Solution (ACD), Pharmacopeia 23 (1995) 119.

[18] S.C.H. Kwok, J. Wang, P.K. Chu, Diamond Relat. Mater. 14 (2005) 78.

[19] E. Chibowski, Adv. Colloid Interf. Sci. 103 (2003) 149.

[20] N.T. Correia, J.J. Moura Ramos, B.J.V. Saramago, J.C.G. Calado, J. Colloid Interf. Sci. 189 (1997) 361.

[21] Interfacial Chemistry, Operating manual DataPhysics OCA, DataPhysics Instruments GmbH, Germany, 2002, pp. 1-4.

[22] ISO 10993-4. Biological Evaluation of Medical Devices. Part 4. Selection of Tests for Interaction with Blood, February 1999.

[23] Y. Imai, Y. Nose, J. Biomed. Mater. Res. 6 (1972) 165.

[24] American Society for Testing and Materials, ASTM F 756-00, Standard Practices for Assessment of Haemolytic Properties of Materials, 2000.

[25] J. Comyn, F. Brady, R.A. Dust, M. Graham, A. Haward, Int. J. Adhesion Adhesives 18 (1998) 51.

[26] W.D. Vilar, Chemistry and Technology of Polyurethanes, Vilar Consultoria, Brazil, 2004 (Chapter 1.2).

[27] J. Comyn, Int. J. Adhesion Adhesives 18 (1998) 247.

[28] S. Venkatraman, R. Gale, Biomaterials 19 (1998) 1119.

[29] S. Agathopoulos, P. Nikolopoulos, J. Biomed. Mater. Res. 29 (1995) 421.

[30] K.S.C.R. Santos, J.F.J. Coelho, P. Ferreira, I. Pinto, S.G. Lorenzetti, E.I. Ferreira, O.Z. Higa, M.H. Gil, Int. J. Pharma. 310 (2006) 37.

[31] P. Ferreira, J.F.J. Coelho, K.S.C.R. Santos, E.I. Ferreira, M.H. Gil, J. Carbohydr. Chem. 25 (2006) 233.

[32] J.F.J. Coelho, A.M.F.P. Silva, A.V. Popov, V. Percec, M.V. Abreu, P.M.O.F Gonçalves, M.H. Gil, J. Polym. Sci.: Part A Polym. Chem. 44 (2006) 2809.

[33] J.F.J. Coelho, A.M.F.P. Silva, A.V. Popov, V. Percec, M.V. Abreu, P.M.O.F Gonçalves, M.H. Gil, J. Polym. Sci.: Part A Polym. Chem. 44 (2006) 3001.

[34] P. Ferreira, J.F. J. Coelho, R. Pereira, António, F.M. Silva, M.H. Gil, J. Appl. Polym. Sci., submitted for publication.

[35] Z. Changsheng, L. Xiangdong, N. Motoyoshi, N. Norio, Biomaterials 24 (2003) 3747. 